\title{
Robust enhancement of high harmonic generation via attosecond control of ionization
}

\author{
Barry D. Bruner ${ }^{1, *}$, Talya Arusi-Parpar ${ }^{1,2}$, Michael Krüger ${ }^{1}$, Oren Pedatzur ${ }^{1}$, Gal Orenstein ${ }^{1}$, \\ Doron Azoury ${ }^{1}$, and Nirit Dudovich ${ }^{1}$ \\ ${ }^{1}$ Department of Physics of Complex Systems, Weizmann Institute of Science, Rehovot 76100 Israel \\ ${ }^{2}$ Applied Physics Department, NRC Soreq, Yavne 81800, Israel
}

\begin{abstract}
We demonstrate up to two orders of magnitude enhancements in high harmonic generation efficiency via sub-cycle control and scaling of the ionization rate in a two colour laser field.
\end{abstract}

\section{Introduction}

Advancements in high harmonic generation (HHG) have led to the development of table-top XUV light sources with applications ranging from ultrafast spectroscopy, X-ray science, and high resolution imaging. However, one of the primary limitations in the application of HHG as a universal XUV source is the low photon flux. Increasing the HHG flux typically involves a careful optimization of the macroscopic aspects of the interaction via phase matching control [1]. While such approaches have successfully enhanced the HHG signal, they all share a common property - the optimization is achieved on a macroscopic level.

In this work we demonstrate a robust enhancement of the HHG yield purely obtained at the microscopic, single particle level [2]. Scaling the enhancement mechanism from the macroscopic to the microscopic regime holds several key fundamental advantages. First, HHG spectroscopy relies on a single particle response, mapping the dynamical properties of the interacting electronic wavefunction into the HHG spectrum. Macroscopic propagation effects can alter the HHG spectral content and phase, preventing accurate reconstructions of the underlying orbital structure and dynamics. Second, the macroscopic schemes require optimization of a number of experimental parameters, among them the laser intensity, wavelength, gas pressure, and interaction length. Here, we present a microscopic scheme that defines a single, easily controllable parameter - namely the ionization probability - to manipulate the HHG yield, therefore providing a robust and scalable approach to circumvent the primary bottleneck in a broad range of HHG applications.

\section{Experiment}

We generated a two colour driving field by temporally and spatially combining beams from a commercial optical parametric amplifier (OPA) (Light Conversion HE-TOPAS-C) and a Ti:S amplifier. Both beams were focused with a spherical mirror into a pulsed gas jet. The HHG emission was measured with a flat field XUV spectrometer consisting of a spherical

\footnotetext{
*e-mail: barry.bruner@weizmann.ac.il
} 
variable line space grating that focuses the harmonics onto a micro channel plate coupled to a phosphor screen, which are then imaged with a CCD camera. Simultaneously, we measured the ionization yield in the gas using a mesh placed $10 \mathrm{~cm}$ from the gas jet, biased at $-300 \mathrm{~V}$, and the HHG photon flux using a Silicon XUV photodiode (Optodiode AXUV100G).

\section{Results}

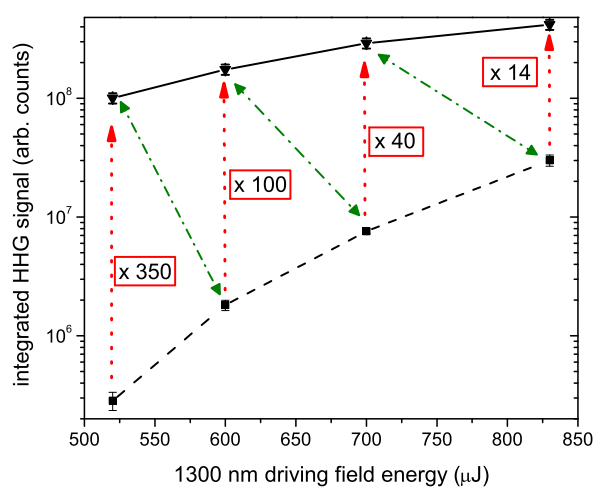

Figure 1. HHG yields in Krypton for single colour fields (dashed black line) and two colour fields (solid black line). The energy in the strong, $1300 \mathrm{~nm}$ field was varied between $520 \mu \mathrm{J}$ and $830 \mu \mathrm{J}$ (corresponding to intensities between $6.3 \times 10^{13} \mathrm{~W} \mathrm{~cm}^{-2}-1.0 \times 10^{14} \mathrm{~W} \mathrm{~cm}^{-2}$ ), while the energy of the weak $800 \mathrm{~nm}$ beam was fixed at $120 \mu \mathrm{J}$ (intensity $2 \times 10^{13} \mathrm{~W} \mathrm{~cm}^{-2}$ ). Red arrows highlight the enhancement factors for each input energy, reaching up to two orders or magnitude $(>300)$. The green dash-dotted lines link the HHG yields, for comparative purposes, from single colour fields and two colour fields with approximately the same total energy.

The interference of the two fields produces a highly modulated pulse in time due to the constructive and destructive interferences between the two carrier waves. Since the ionization rate depends exponentially on the total field strength, the number of ions - and HHG photons - is boosted considerably in the regions of constructive interference. A complete analysis of the subcycle dynamics appears in our recent work [2]. These concepts are general and have been applied toward HHG enhancement in a number of atomic and molecular gases $(\mathrm{Kr}, \mathrm{Ar}$, $\mathrm{Ne}, \mathrm{He}, \mathrm{N}_{2}, \mathrm{CO}_{2}$ ) and a range of fundamental wavelengths of the strong driving field.

Systematic measurements of the HHG yield in Krypton for single colour (1300 nm only) and two colour $(1300+800 \mathrm{~nm})$ fields are shown in Fig. 1. Despite the small increases in total input energy from the weaker $800 \mathrm{~nm}$ field (varying between 14 and $23 \%$ ), the corresponding enhancement in HHG yield can reach over two orders of magnitude ( $>300$ ). We also note the significant differences between the HHG yields from single colour fields and two colour fields with approximately the same total energy (dashed green arrows in Fig. 1). This observation demonstrates that the enhancement mechanism is not induced by the increase in the total input energy of the driving field - it is a direct outcome of the two fields interference, manipulating the interaction on a subcycle scale.

In the following step we directly map the linkage between ionization probability and HHG yield by tracking both signals at the single shot level. For HHG driven by multicolour fields where the fields are not phase locked, the output spectrum for laser shot represents a different realization of their relative phase [3]. Various sources of instability such as gas density fluctuations can also cause variations in the HHG and ion signals. A single shot analysis 


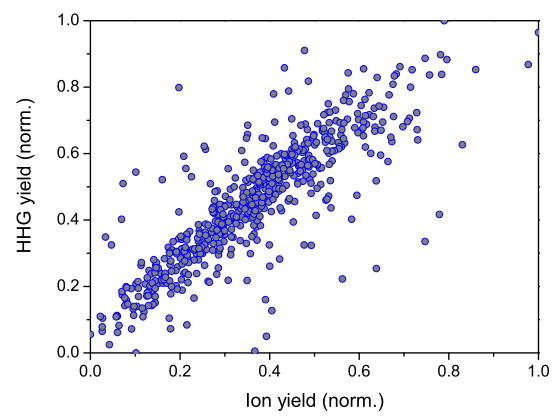

Figure 2. Scatter plot of single shot HHG and ion yields. The energies (intensities) of the $1300 \mathrm{~nm}$ and $800 \mathrm{~nm}$ beams were $600 \mu \mathrm{J}\left(7.3 \times 10^{13} \mathrm{~W} \mathrm{~cm}^{-2}\right)$ and $120 \mu \mathrm{J}\left(2 \times 10^{13} \mathrm{~W} \mathrm{~cm}^{-2}\right)$ respectively. The yields were normalized on a zero to one scale between the minimum and maximum recorded values. The correlation degree is 0.84 .

allows us to map a specific XUV spectrum to its corresponding ionization yield - information that is hidden by signal averaging. Fig. 2 displays a scatter plot between the single shot ion and HHG yields, for a sequence of 600 laser shots. Importantly, we observe a strong correlation as well as a strict linear dependence between them. This observation explicitly links the HHG yield with the microscopic, single particle response of the atoms with respect to the laser field. Macroscopic phase matching effects are minimized by producing HHG over a short interaction length in the jet, such that the macroscopic field scales as the microscopic emission. Any additional, macroscopic response would give a strict deviation from this linearity. Phase matching calculations (not shown) support this interpretation [2].

\section{Conclusions}

This work demonstrates the feasibility of energy scalable HHG sources based on subcycle control and enhancement of ionization. Recently, we have extended this work toward efficient production of HHG-based soft X-ray light [5]. This arrangement is also highly favourable for HHG spectroscopy. As new HHG spectroscopy paradigms emerge for studying increasingly complex molecules [6], it is essential to understand and manipulate the response at the single molecule level. Enhancing a single parameter - the ionization yield - will enable us in future research to identify and isolate the HHG contribution of inner orbitals. Revealing the contributions of these orbitals, commonly hidden in many HHG experiments due to their low ionization yield, will resolve rich multielectron, attosecond scale, phenomena.

\section{References}

[1] T. Popmintchev et. al., Nat. Photonics 4, 822-832 (2010).

[2] B. D. Bruner, M. Krüger, O. Pedatzur, G. Orenstein, D. Azoury, and N. Dudovich, Opt. Express 26, 9310-9322 (2018).

[3] E. J. Takahashi, P. Lan, O. D. Mücke, Y. Nabekawa, and K. Midorikawa, Nat. Commun 4, 2691 (2013).

[4] Y. Pertot et. al., Science 355, 10.1126/science.aah6114 (2017).

[5] B. D. Bruner et. al., in preparation.

[6] B. D. Bruner et. al., Faraday Discuss 194, 369-405 (2016). 